\title{
Claves para descifrar el sistema imperial de innovación comandado por Estados Unidos
}

\section{Raúl Delgado Wise*}

Resumen. Un rasgo consustancial del capitalismo contemporáneo es la concentración y apropiación privada sin precedentes de los productos del General Intellect a través del patentamiento. Se trata de un fenómeno que atraviesa todos los poros de la sociedad capitalista y que, lejos de favorecer una ruta progresista de desarrollo de las fuerzas productivas, conduce a una fase regresiva en el avance del conocimiento, que profundiza el desarrollo desigual y atenta contra la naturaleza y la humanidad entera, a grado tal de convertirse en una amenaza de dimensiones y alcances civilizatorios. Este trabajo se propone contribuir en el desentrañamiento de la nueva arquitectura que caracteriza a los sistemas de innovación en la actualidad y que ha dado lugar a lo que aquí se concibe como un sistema imperial de innovación, con epicentro en Silicon Valley y su constelación de satélites a lo largo y ancho del planeta.

Palabras clave: sistema imperial de innovación, patentes, capitalismo neoliberal, Organización Mundial de la Propiedad Intelectual, apropiación del conocimiento.

* Director de la Unidad Académica de Estudios del Desarrollo de la Universidad Autónoma de Zacatecas, México. 


\section{Keys for deciphering the imperial innovation system led by the United States}

Abstract. One key trait of contemporary capitalism is the unprecedented concentration and private accumulation of the products of General Intellect through the patenting system. This phenomenon seeps through all the pores of capitalist society and — far from encouraging a progressive development path for the forces of production - drives a regressive phase in the advancement of knowledge that deepens unequal development and threatens nature and the whole of humanity, to the degree that it is a threat of immense scale and reach. This study intends to contribute to the unravelling of the new architecture that characterizes current innovation systems and which have given way to that which we concieve of as an imperial system of innovation, with its epicenter in Silicon Valley and its constellation of satellites which ring the entire planet.

Keywords: imperial system of innovation, patents, neoliberal capitalism, World Intellectual Property Organization, knowledge appropriation. 


\section{Introducción}

Asistimos a una época en la que las patentes se han convertido en un aspecto consustancial a la lógica de reestructuración capitalista promovida por las grandes corporaciones multinacionales y los gobiernos imperialistas encabezados por Estados Unidos. Este fenómeno se produce en el marco de lo que Samir Amin (2013) concibe, con perspicacia, como la era de los monopolios generalizados. Los desbordantes niveles conseguidos por la concentración y centralización del capital han alterado el modus operandi propio de las esferas de producción, circulación y distribución del capital, lo que acentúa a grados extremos las contradicciones del sistema. Un aspecto clave y relativamente poco estudiado del proceso es la profunda reestructuración que experimentan los sistemas de innovación desde la década de 1990 y, con particular ímpetu, a partir del siglo XXI. En esos años la expansión, la concentración y la apropiación privada de los productos del General Intellect han alcanzado categorías y ritmos inimaginables, que penetran todos los poros de la sociedad capitalista y que, lejos de propiciar una ruta progresista de desarrollo de las fuerzas productivas, promueven una fase regresiva y oscurantista en el avance del conocimiento. Ello profundiza el desarrollo desigual y atenta contra la naturaleza y la humanidad entera, a tal punto que se convierte en una amenaza de dimensiones y alcances civilizatorios.

En efecto, en el curso de las últimas dos décadas y media se han producido drásticas transformaciones en las formas de generación y apropiación del conocimiento. Entre otros aspectos, las dinámicas de apropiación del trabajo científico-tecnológico por la vía del patentamiento se han expandido y crecido a ritmos sin parangón en la historia del capitalismo 
contemporáneo. Se trata, en el fondo, de un viraje transcendental en la forma de operación y desarrollo de los sistemas de innovación, con epicentro en Estados Unidos, y cuyos artífices primordiales son las grandes corporaciones multinacionales. Una parte significativa de la lógica que subyace en los acuerdos de libre comercio en boga, tales como el Tratado de Libre Comercio de América del Norte (TLCAN), la Asociación de Naciones del Sudeste Asiático (ASEAN, por sus siglas en inglés) y el Acuerdo Transatlántico de Cooperación Económica (TTP, por sus siglas en inglés), reside en este proceso y en el tejido institucional de raigambre neoliberal que lo sustenta, impulsado por el Banco Mundial (BM), el Fondo Monetario Internacional (FMI), la Organización Mundial del Comercio (OMC) y, más recientemente - como pieza clave de las nuevas dinámicas de innovación y apropiación de los productos del trabajo científico-tecnológico-, la Organización Mundial de la Propiedad Intelectual (WIPO, por sus siglas en inglés).

Al interior del argumento aquí presentado subyace la hipótesis de que los sistemas de innovación atraviesan por una profunda fase de reestructuración comandada por las grandes corporaciones multinacionales, sobre todo de origen estadounidense - con epicentro en Silicon Valley y sus satélites alrededor del mundo-, la cual da lugar a una desbordante carrera por la generación y concentración de patentes. Bajo el nuevo andamiaje, que al lema «iacumulad, acumulad!», agrega la proclama de «ipatentad, patentad!», el capital monopolista, en su insaciable afán de lucro, torna en su contrario el carácter progresista que Marx atribuyó al desarrollo de las fuerzas productivas de la sociedad durante el capitalismo: una senda que atenta contra la naturaleza y contra la vida misma. Lo anterior no sólo cuestiona la noción de desarrollo sino que promueve la generación de nuevos modos de intercambio desigual que acentúan las asimetrías entre países, agudizan las 
desigualdades sociales, al tiempo que desajustan los mecanismos del sistema y lo sumergen en una profunda crisis que amenaza a la humanidad entera y de la que en apariencia no ha podido encontrar más que falsas salidas; es el caso de la financiarización, el extractivismo, el acaparamiento de tierras (land grabbing) y la sobreexplotación salarial.

\section{A manera de contextualización: radiografía del capital monopolista}

Un primer rasgo omnipresente en el capitalismo contemporáneo es el hecho de que, como en ninguna otra época de su larga historia, el capital monopolista se ha convertido en el agente dominante de la economía política internacional. Su influencia es tal que, como se indicó al comienzo, Samir Amin (2013) se refiere a la época actual como la de los monopolios generalizados. A través de megafusiones y alianzas estratégicas, esta fracción del capital ha llegado a niveles de concentración y centralización inimaginables: «Las mayores compañías del mundo (aquellas con más de mil millones [de dólares estadounidenses] en ventas anuales) (...) dan cuenta de aproximadamente 60 por ciento del ingreso, 65 por ciento de la capitalización de mercado y 75 por ciento de las ganancias [mundiales]» (McKinsey Global Institute, 2015:21). No es, empero, un simple cambio cuantitativo, sino una profunda transformación cualitativa en las formas de organización y dominio del capital monopolista basadas en la financiarización, el saqueo de recursos naturales de los países periféricos y las ventajas comparativas derivadas del arbitraje laboral global, es decir, la persistencia de significativos diferenciales salariales entre países y regiones. 
En esencia, comprende una reestructuración del capital monopolista a escala planetaria, la cual ha dotado al imperialismo contemporáneo de un nuevo rostro (Suwandy y Foster, 2016) que se caracteriza, entre otros elementos, por:

1. La financiarización vinculada al ascenso y predominio del capital financiero sobre otras fracciones del capital (Bello, 2005). Ante la falta de inversiones redituables en la esfera productiva por la crisis de sobreproducción desencadenada a fines de la década de 1970, el capital comienza a trasladarse hacia la especulación financiera (Brenner, 2002). Asimismo, con la presión «a la baja» ejercida en los salarios reales mediante el arbitraje laboral global, se promueve una explosión de deudas encabezada por el sector financiero, la cual posibilita que la producción encuentre canales de realización, aunque sean endebles e insustentables. Ocurre así la financiarización de la clase capitalista, del capital industrial y de las ganancias corporativas, que impulsa una explosión de capital ficticio, de títulos financieros sin contraparte en la producción material (Foster, 2010). En opinión de Sergio Rodríguez Lascano (2015), implica una mutación del ciclo básico del capital D-M-D’ en D-D'.

2. La configuración de redes globales de capital monopolista generadas a partir de una estrategia de reestructuración encabezada por las grandes corporaciones multinacionales que, por medio de operaciones de outsourcing y cadenas de subcontratación, extienden segmentos de sus procesos productivos, comerciales, financieros y de servicios a los países periféricos en busca de mano de obra flexible y barata. Un claro ejemplo de esa estrategia son las plataformas de exportación que operan como economías de enclave en los países periféricos.

El giro hacia lo que algunos autores, entre ellos Gary Gereffi y Timothy Sturgeon (2013), caracterizan como cadenas globales de valor ha sido 
espectacular: «Las 100 mayores corporaciones globales han desplazado su producción hacia sus filiales extranjeras [en concreto en el Sur], donde ahora se localizan cerca de 60 por ciento del total de sus bienes y empleados y más de 60 por ciento de sus ventas a nivel global» (UNCTAD, 2010). En realidad entraña un «nuevo «nomadismo» surgido al interior del sistema de producción global, en el que la selección de localidades se determina en buena parte a partir de dónde es más barata la mano de obra» (Foster et al., 2011a:18). En esa perspectiva, cabe destacar que: a) al menos 40 por ciento del comercio mundial se asocia a operaciones de outsourcing, incluyendo subcontrataciones y comercio intrafirma entre filiales de una misma compañía (Andreff, 2009); y b) se estima que en la periferia capitalista hay 85 millones de trabajadores directamente empleados en más de 3 mil 500 zonas de procesamiento para la exportación ubicadas en 130 países (McKinsey Global Institute, 2012).

La estrategia de reestructuración en cuestión ha modificado la geografía global de la producción; en la actualidad poco más de 70 por ciento del empleo industrial se localiza en países periféricos (Foster et al., 2011b). Lo significativo de la estrategia es que descansa en el aprovechamiento y la profundización de las brechas salariales entre países y regiones; esto es, el llamado arbitraje laboral global que para autores como Intan Suwandi y John Bellamy Foster (2016) constituye el rasgo distintivo del imperialismo contemporáneo. Bajo tal mecanismo, las grandes corporaciones multinacionales logran obtener ganancias extraordinarias con el establecimiento de límites a la libre movilidad de la más importante mercancía para la acumulación de capital: la fuerza de trabajo (Delgado y Martin, 2015).

3. El extractivismo y el nuevo extractivismo (Gudynas, 2015) en alusión a la creciente apropiación y exportación de minerales, petróleo y gas de 
los países periféricos por las grandes corporaciones extractivas nacionales y multinacionales, a través de la sobreexplotación de recursos naturales y la consecuente expropiación de bienes comunes, lo que exacerba los conflictos sobre territorios y aguas (Veltmeyer, 2013). Ello ha traído consigo severas consecuencias para el medio ambiente a causa de la minería a tajo o cielo abierto, el fracking, el gas lutitas, etcétera.

Dado que los ingresos de varias de las corporaciones multinacionales más poderosas del mundo dependen de la extracción, la producción y el consumo de combustibles fósiles, lo más probable es que este patrón persista, situación que ahondará aún más la crisis ecológica y sus efectos sobre el calentamiento global y el cambio climático. La nueva ola extractivista originada por la reestructuración neoliberal ha empeorado la degradación ambiental, no sólo al expandir la geografía de la destrucción, también con la estrategia del arbitraje ambiental regulatorio de parte del capital extractivo (Xing y Kolstad, 2002).

4. El acaparamiento o control de la tierra y recursos naturales por los agronegocios (land grabbing). Este fenómeno se relaciona a cambios en los regímenes alimentarios acompañados de dinámicas de financiarización, procesos de acumulación por desposesión (que han implicado un brutal despojo de campesinos y pueblos originarios), alteraciones en los patrones de cultivo (monocultivo, uso de transgénicos, etcétera) y daños severos e irreversibles al entorno natural (pérdida de biodiversidad, destrucción de suelos, quebranto de la soberanía alimentaria, etcétera) (Borras et al., 2012). En este caso, se produce también una notable expropiación de bienes comunes, con la consecuente exacerbación de los conflictos sobre territorios y aguas.

El otro efecto de la profunda reestructuración y el espectacular crecimiento de la concentración y centralización del capital monopolista 
es, precisamente, la brutal embestida desencadenada en contra de la clase trabajadora y los sectores populares en todos los rincones del planeta. En ese sentido, otra pieza fundamental del imperialismo contemporáneo es la reconfiguración de la división internacional del trabajo, en la que la fuerza de trabajo figura de manera abierta, a tono con la perspectiva marxista planteada por Emmanuel (1972) para analizar el intercambio desigual, como la principal mercancía de intercambio entre países centrales y periféricos, lo que ocasiona la aparición de nuevas y extremas formas de intercambio desigual (Márquez y Delgado, 2011). La dinámica de creciente internacionalización de las finanzas, la producción, el comercio y los servicios, propia del capitalismo neoliberal, se acompaña de una creciente fragmentación de la geografía mundial y un desbordante aumento de las desigualdades sociales a niveles inusitados hasta hace poco:

Tan sólo ocho personas (ocho hombres en realidad) poseen ya la misma riqueza que 3 mil 600 millones de personas, la mitad más pobre de la humanidad. La súper concentración de riqueza sigue imparable. El crecimiento económico tan sólo está beneficiando a los que más tienen. El resto, la gran mayoría de ciudadanos de todo el mundo y especialmente los sectores más pobres, se están quedando al margen de la reactivación de la economía. El modelo económico y los principios que rigen su funcionamiento nos han llevado a esta situación que se ha vuelto extrema, insostenible e injusta. Es hora de plantear una alternativa (Oxfam, 2017:1).

Este «orden» o desorden planetario encierra profundas y peligrosas contradicciones (Harvey, 2014) que desde la década de 1970 han sumergido al capitalismo mundial en una crisis aguda que abrió la puerta a la 
implantación del neoliberalismo y que persiste hasta la fecha. En efecto, la Hidra capitalista con sus nuevos rostros —en alusión a la extraordinaria metáfora capitalista que alude al régimen del capital— sólo ha encontrado falsas y limitadas salidas a la crisis que le dio origen y, peor aún, lejos de abrir caminos hacia una fase sostenida de crecimiento de la economía mundial, a cada paso ha contribuido a profundizarla y a la tormenta. En palabras de Humberto Márquez (2010:67):

La crisis que afronta el capitalismo contemporáneo representa una ruptura en el proceso de expansión capitalista promovido por los países centrales, encabezados por Estados Unidos, desde la década de los setenta. Se trata de una reestructuración fallida que ha hecho colapsar al centro mismo del sistema capitalista mundial, sobre todo a sus centros financieros e industriales más dinámicos, y que se ha transmitido con prontitud hacia todos los sectores, circuitos y rincones del capitalismo. Sin embargo, no podemos perder de vista que la estrategia de reestructuración y expansión ha cumplido con creces su cometido principal: concentrar capital, poder y riqueza en manos de una delgada elite de capitalistas transnacionalizados y, en contrapartida, ha deteriorado de manera drástica las condiciones de vida y trabajo de la mayoría de la población.

Por consiguiente, nos hallamos en una grave crisis multidimensional: financiera, económica, social, cultural y ecológica, que no sólo ha puesto en jaque al sistema capitalista, sino que al poner en predicamento las fuentes materiales de la vida — el trabajo y la naturaleza — ha adquirido proporciones civilizatorias. En el trasfondo de esta compleja e intrincada trama subyace una quinta dimensión de la metamorfosis experimentada 
por el capital monopolista: la vasta reestructuración operada en los sistemas de innovación a partir de la década de 1990, la cual es fundamental en la comprensión de las contradicciones esenciales que caracterizan al capitalismo contemporáneo.

\section{Claves para descifrar la reestructuración de los sistemas de innovación}

Las formas tradicionales de comprender los sistemas de innovación, como la triple hélice o el triángulo de Sábato, resultan anacrónicas e inapropiadas para desentrañar las nuevas dinámicas de «desarrollo» científico y tecnológico. Hablar de sistema nacional de innovación es igualmente inadecuado para captar la complejidad de los ecosistemas dominantes. Al respecto, tomando como referente el caso paradigmático de Silicon Valley y sus satélites, es posible distinguir cuatro ejes que, en grandes pinceladas, describen las dinámicas de generación y apropiación del trabajo científico-tecnológico promovidas bajo la égida del capitalismo neoliberal (Delgado, 2015).

a) Internacionalización y fragmentación de las actividades de investigación y desarrollo en alusión a la preeminencia de modalidades «colectivas»: peer-to-peer, share economy, commons economy y crowdsourcing economy de innovación, a través de lo que se conoce como innovación abierta (open innovation). Son modalidades de invención «extramuros», fuera del entorno de la corporación multinacional, que entrañan una apertura y redistribución espacial de actividades intensivas en conocimiento, con la creciente participación de socios externos a las grandes corporaciones multinacionales 


\section{RAÚl DELGADO WISE}

(start-ups, proveedores de capital de riesgo, clientes, subcontratistas, head hunters, firmas de abogados, universidades y centros de investigación), lo que ha fomentado la permanente configuración y reconfiguración de redes de innovación (véase figura 1).

Figura 1. Representación gráfica del ecosistema de Silicon Valley

Stanford, University of California

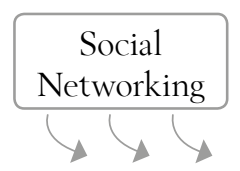

(Berkeley), University of San Francisco,

San Francisco State University, San Jose

State University, University of California

(Santa Cruz), Santa Clara University

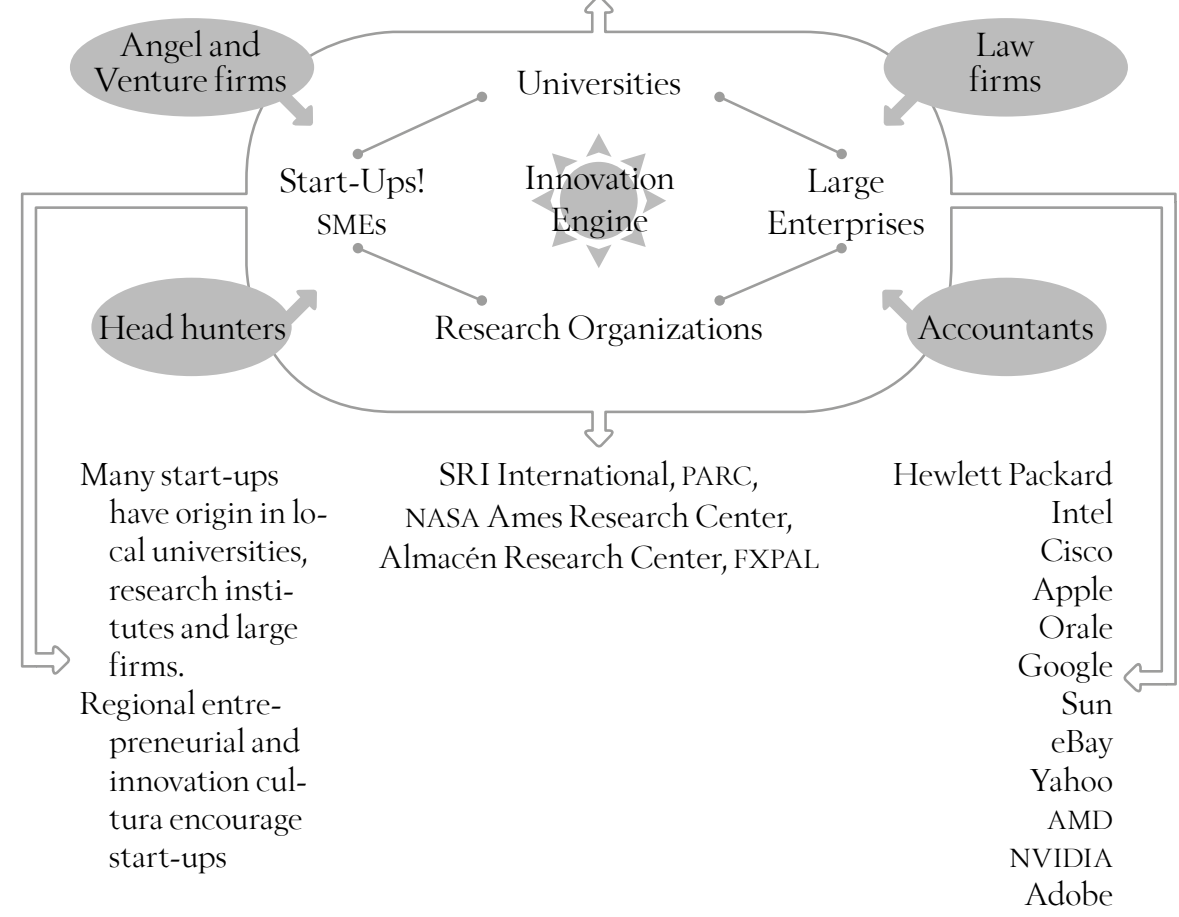

Fuente: Strategic Business Insights. 
b) Ciudades cientificas, por medio de las cuales se crean sinergias colectivas que conducen a una aceleración de la investigación y el desarrollo y, en consecuencia, de la innovación. Consiste, de acuerdo con AnnaLee Saxenian (2002 y 2006), en un nuevo paradigma que se aparta de los viejos modelos «cerrados» de investigación y desarrollo, y hace factible una nueva cultura de la innovación basada en la flexibilidad, la descentralización y la incorporación, bajo diferentes modalidades, de nuevos y cada vez más numerosos jugadores que interaccionan en espacios locales y transnacionales. Silicon Valley figura como el pivote de una nueva arquitectura de la innovación mundial, a cuyo alrededor se tejen múltiples eslabones periféricos que operan como una suerte de maquiladoras científicas localizadas en regiones, ciudades y universidades de todo el mundo.

c) Nuevas formas de control de las agendas de investigación y de apropiación de los productos del trabajo científico —es decir las patentespor las grandes corporaciones multinacionales (CMN) a través de multivariadas formas de subcontratación, asociación, manejo y diversificación del capital de riesgo. Y lo más importante, la disposición de equipos especializados de abogados que negocian con los potenciales subcontratistas y operan a la manera de head hunters, pues conocen los entretelones de la operación de la nueva y compleja arquitectura de los sistemas de patentamiento, establecidos en el Tratado de Cooperación sobre patentes de la WIPO (véase figura 2). A las nuevas formas de gestión y control de las agendas de innovación y apropiación de patentes (véase figura 3) se les conoce como strategic investment (Galama y Josek, 2008). 


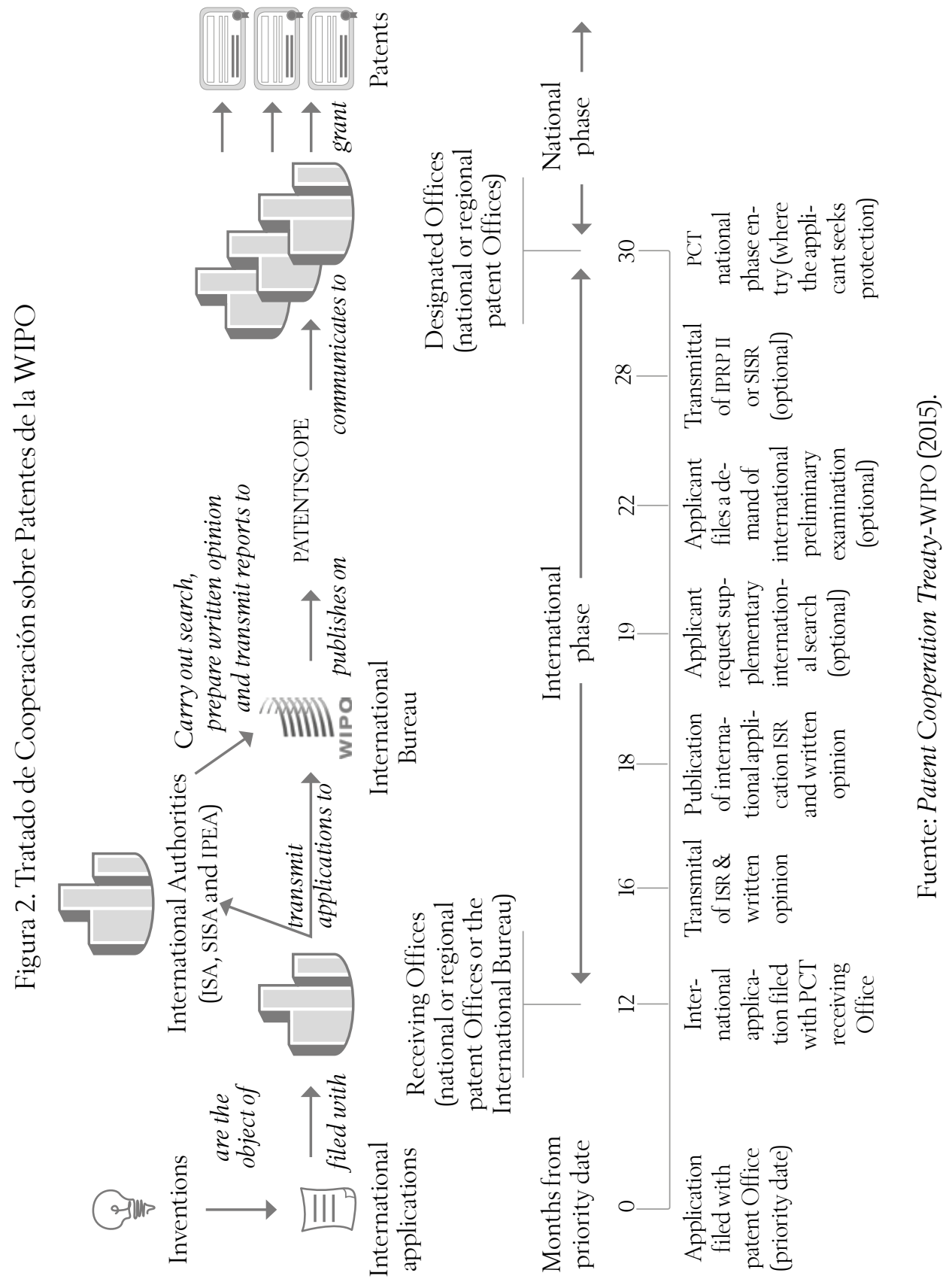


Figura 3. Solicitudes de patentes según tipo de solicitante PCT-WIPO* 1996-2010
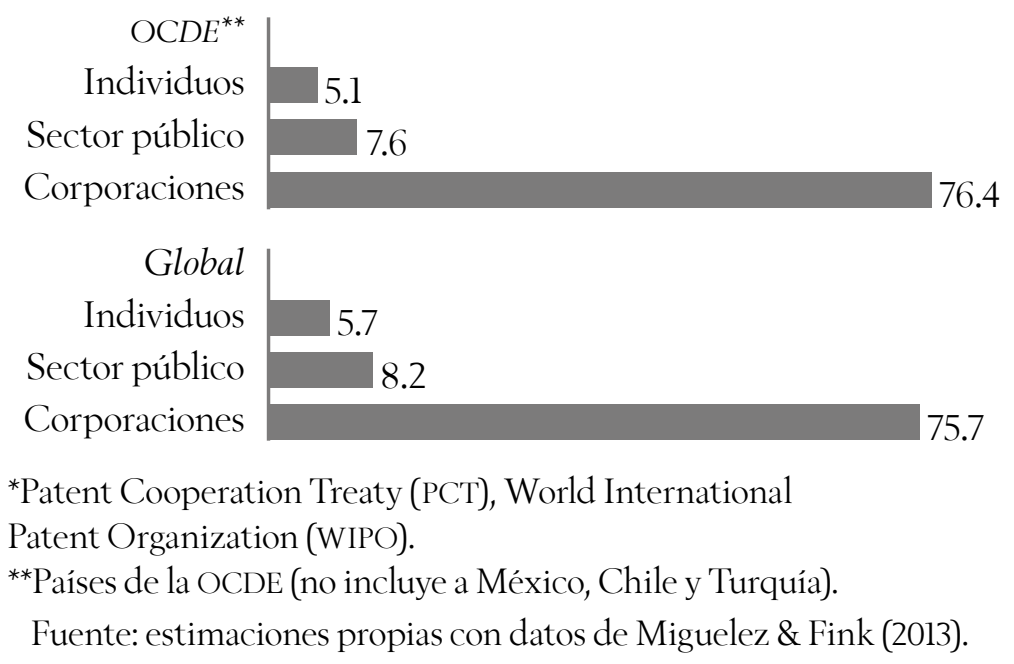

d) Expansión en el horizonte Norte-Sur de la fuerza de trabajo en áreas de ciencia, tecnología, innovación y matemáticas y el creciente reclutamiento de fuerza de trabajo altamente calificada proveniente de las periferias vía outsourcing y offshoring. La migración altamente calificada desempeña un papel cada vez más relevante en los procesos de innovación, circunstancia que propicia una paradójica y contradictoria dependencia del Sur respecto del Norte: cada vez más los generadores de patentes son originarios de países periféricos; según datos de la WIPO, en 2014 aproximadamente la mitad provenía de esas latitudes, no obstante que alrededor de 75 por ciento de las patentes fueron concentradas y apropiadas por corporaciones multinacionales (véase figura 3).

Teniendo en cuenta este nuevo escenario, que perfila lo que podría concebirse como la implantación de un sistema imperial de innovación, 
a continuación se describirá la estrategia en materia de derechos de propiedad intelectual seguida por Estados Unidos a partir de 1990. No debe perderse de vista — atendiendo a lo reseñado en este apartadoque las patentes encierran una permanente y creciente tensión entre los beneficios de las grandes corporaciones, los intereses de los innovadores y las necesidades de los consumidores y de la sociedad en general. En dicha tensión es, precisamente, donde el «derecho internacional» impuesto por los organismos internacionales al servicio de Estados Unidos devela, sin ambages, su carácter eminentemente parcial a favor de las primeras.

\section{Las patentes como instrumentos de apropiación del General Intellect}

Desde finales de la década de 1980, en Estados Unidos inició una tendencia a legislar ad hoc, de conformidad con los intereses estratégicos de las grandes corporaciones concernientes a la propiedad intelectual (Messitte, 2012). Con las regulaciones impulsadas por la Organización Mundial del Comercio (OMC) - Acuerdo sobre los Aspectos de los Derechos de Propiedad Intelectual relacionados con el Comercio (Díaz, 2008; Guerrero y Gutiérrez, 2011) — dicha normatividad se expandió a escala global durante las negociaciones para la suscripción y puesta en marcha de los Tratados de Libre Comercio (TLC) (García, 1998). Las negociaciones se realizaron a través de la Oficina del Representante Comercial de Estados Unidos, quien a su vez ha protegido y representado los intereses de las industrias intensivas en el uso de la propiedad intelectual y farmacéutica, Business Software Alliance (BSA) en derechos de autor y la Pharmaceutical Research 
and Manufacturers of America (PhRMA) en patentes farmacéuticas (Díaz, 2008). ${ }^{1}$ Por su carácter multilateral las disputas acerca de propiedad intelectual al seno de la OMC tienden a ser complejas, de ahí que la estrategia estadounidense contemple asimismo la negociación bilateral de TLC, a fin de controlar mercados y acrecentar ganancias corporativas.

Dentro de los procesos de intercambio comercial, un aspecto medular de los TLC ha sido favorecer a los inversionistas (léase, las grandes corporaciones multinacionales), mediante la extensión y ampliación de la cobertura de patentes y la flexibilización de requisitos de patentamiento, dejando de lado las funciones que, en principio, debieran cumplir las propias patentes: asegurar que la creación cumpla con los requisitos de invención, originalidad y aplicabilidad socioeconómica, así como difundir el conocimiento incorporado en la invención y evitar con ello la duplicación de esfuerzos (Díaz, 2008). En otras palabras, los TLC atentan contra la naturaleza de bien común que debiera tener el conocimiento, en tanto motor del progreso y desarrollo de las fuerzas productivas de la sociedad.

\section{Impactos de los acuerdos comerciales}

En primer lugar, uno de los impactos importantes - y no tan obvio- es la adhesión de países periféricos a los convenios relacionados con patentes, ${ }^{2}$

${ }^{1}$ Estas industrias enfrentan altos costos de investigación y desarrollo (I+D), mientras que el costo marginal de reproducción de los nuevos bienes es muy reducido; las patentes les proveen de una ventaja competitiva de mercado.

${ }^{2}$ Cabe apuntar que parte de la agenda del TPP por el convenio bilateral Estados Unidos-Unión Europea es penetrar en los mercados internos de varios países de la Unión Europea, donde el arribo de las grandes corporaciones multinacionales ha sido contenido mediante normas internas de protección. 
además de la adecuación interna de sus leyes sobre propiedad intelectual. Esto ha ocurrido sin que tales modificaciones guarden conexión alguna con el nivel interno de desarrollo de la ciencia y la tecnología. En opinión de Shadlen (2011:68), «México adoptó un sistema de patentes que es apropiado para un país con infraestructura científica, tecnológica e industrial significativamente más avanzada». Lo anterior cobra mayor sentido aún si se considera que la generación de patentes se vincula con el gasto en investigación y desarrollo, el tipo de inversión que se lleva a cabo en investigación científica aplicada o innovación patentable y la capacidad de absorción de conocimientos tecnológicos (Díaz, 2008). A contrario sensu, si no operara la lógica corporativa e imperialista referida, la legislación correspondiente a propiedad intelectual debería ser diametralmente distinta, lo que permitiría a los países periféricos desarrollar sus capacidades endógenas en ciencia, tecnología e innovación.

En segundo lugar, entre 1991 y 2011 se promovió una dinámica desbordante de crecimiento en el plano del patentamiento, sintetizada en el lema antes referido y por lo demás ajeno a una visión positiva y equitativa del progreso: «ipatentad, patentad!» En efecto, como se aprecia en la figura 4, en las dos décadas referidas se generaron en Estados Unidos más patentes que en 300 años de historia previa. Este desproporcionado y espectacular crecimiento, aunque se asocia estrechamente con la lógica de la reestructuración de los sistemas de innovación descrita en el primer apartado, entraña también una cierta ampliación, vulgarización y aprisionamiento (enclosure) de lo que materialmente tiene sentido patentar y que daña la lógica del conocimiento como bien común, en tanto producto del General Intellect conforme a la definición de Marx de trabajo científico. Más aún, en ocasiones se trata de patentes adquiridas con la finalidad 
de no aplicarlas o postergar su aplicación para que el capital monopolista controle y regule los mercados, dando lugar a lo que Guillermo Foladori (2014) concibe como «ciencia ficticia» por su carácter especulativo, que semeja la noción acuñada por Marx de capital ficticio.

Figura 4. Evolución de las patentes registradas en Estados Unidos

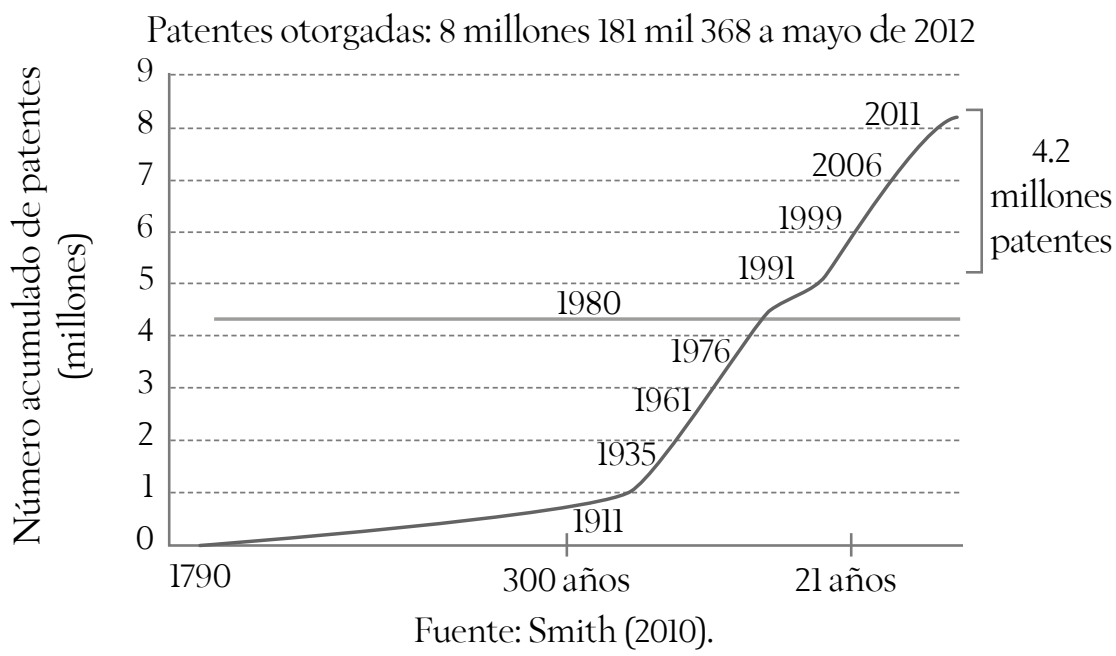

A tono con lo hasta aquí referido, del cuadro 1 se desprende que Estados Unidos despunta como la primera potencia capitalista del mundo en materia de innovación, ${ }^{3}$ al concentrar 28 por ciento de la totalidad de patentes gestionadas. Al considerar al total de países de la OCDE — sin incluir a México, Chile y Turquía- se aprecia que, en conjunto, concentran 90

${ }^{3}$ En sintonía con su dominio en el plano de las patentes, Estados Unidos sigue siendo la primera potencia imperialista del planeta, como se ha planteado a lo largo de este trabajo, al dominar también en el área de las corporaciones multinacionales, la moneda mundial, la fuerza militar y al ejercer control sobre los principales organismos internacionales: BM, FMI, OMC y WIPO. 
por ciento de las solicitudes mundiales de patentes. Asimismo, se observa un bajo nivel de participación de América Latina y el Caribe en la generación de patentes, cuyo aporte al cúmulo global de solicitudes consignadas fue de apenas 0.7 por ciento.

\section{Cuadro 1}

Solicitudes de patentes, PCT-WIPO* 1996-2010

\begin{tabular}{|c|c|c|c|}
\hline Pais & $\begin{array}{c}\text { Solicitudes } \\
\text { patentes }\end{array}$ & País & $\begin{array}{c}\text { Solicitud } \\
\text { patentes }\end{array}$ \\
\hline Total global & 4482343 & & \\
\hline Total OCDE ${ }^{* *}$ & 4032186 & América Latina y el Caribe & 30366 \\
\hline Primeros 10 países & 3673953 & $\begin{array}{l}\text { Primeros seis países } \\
\text { de América Latina } \\
\text { y el Caribe }\end{array}$ & 26778 \\
\hline Estados Unidos & 1237060 & & \\
\hline Japón & 710516 & Brasil & 12779 \\
\hline Alemania & 627460 & México & 6335 \\
\hline Reino Unido & 216480 & Argentina & 2966 \\
\hline Francia & 212571 & Colombia & 1673 \\
\hline China & 208665 & Chile & 1522 \\
\hline República de Corea & 183584 & Cuba & 1503 \\
\hline Canadá & 102917 & & \\
\hline Países Bajos & 93105 & & \\
\hline Suecia & 81595 & & \\
\hline
\end{tabular}

*Patent Cooperation Treaty (PCT), World International

Patent Organization (WIPO).

${ }^{* *}$ No incluye a México, Chile y Turquía.

Fuente: estimaciones propias con datos de Miguelez \& Fink (2013). 
Cuadro 2

Solicitud de patentes: balance en el horizonte Norte-Sur (1990-2010)

\begin{tabular}{|c|c|c|c|c|c|c|c|}
\hline \multirow{2}{*}{$\begin{array}{l}\text { Dirección } \\
\text { de flujos }\end{array}$} & \multirow{2}{*}{$\begin{array}{l}\text { Periodo } \\
1990-2010\end{array}$} & \multicolumn{2}{|c|}{$A \tilde{n} o$} & \multirow{2}{*}{$\begin{array}{c}\text { Tasa de } \\
\text { crecimiento } \\
1990-2010\end{array}$} & \multicolumn{3}{|c|}{$\begin{array}{c}\text { Distribución } \\
\text { porcentual }\end{array}$} \\
\hline & & 1990 & 2010 & & $1990-2010$ & 1990 & 2010 \\
\hline Total & 820072 & 2922 & 91720 & 18.8 & 100.0 & 100.0 & 100.0 \\
\hline $\begin{array}{l}\text { Sur-Norte } \\
\text { países } \\
\text { no OCDE } \\
\text { a países } \\
\text { OCDE* }^{*}\end{array}$ & 317946 & 654 & 39936 & 22.8 & 38.8 & 22.4 & 43.5 \\
\hline $\begin{array}{l}\text { Norte-Sur } \\
\text { países OCDE } \\
\text { a países } \\
\text { no OCDE* }\end{array}$ & 23598 & 54 & 3822 & 23.7 & 2.9 & 1.8 & 4.2 \\
\hline $\begin{array}{l}\text { Norte-Norte } \\
\text { países OCDE } \\
\text { a países } \\
\text { OCDE* }^{*}\end{array}$ & 464900 & 2208 & 45880 & 16.4 & 56.7 & 75.6 & 50.0 \\
\hline $\begin{array}{l}\text { Sur-Sur } \\
\text { países } \\
\text { no OCDE } \\
\text { a países } \\
\text { no OCDE* }\end{array}$ & 13628 & 6 & 2082 & 34.0 & 1.7 & 0.2 & 2.3 \\
\hline
\end{tabular}

Patent Cooperation Treaty (РCT), World International Patent Organization

(WIPO). * No se incluye a México, Chile y Turquía.

Fuente: estimaciones propias con datos de Miguelez \& Fink (2013)

y países firmantes del PCT (WIPO, 2017).

Tras este aumento acelerado de las solicitudes de patentes se registra, por un lado, un incremento de las solicitudes extranjeras en países periféricos. Así, en el caso de México, con la entrada en vigor del TLCAN 
se da un proceso de sustitución de patentes nacionales por extranjeras (Díaz, 2008). Por otro lado, y de manera un tanto paradójica, en la Oficina de Patentes y Marcas Registradas de Estados Unidos se percibe una tendencia hacia el aumento de solicitudes de extranjeros, principalmente provenientes de países del Sur.

En tercer lugar, es en la industria farmacéutica donde se presentan los efectos más visibles de las legislaciones aprobadas con relación a quiénes ganan y quiénes pierden. Los ganadores son las grandes corporaciones multinacionales, con dividendos provenientes de mercados monopólicos y de compensaciones por atrasos «injustificados» en la tramitación de patentes y permisos de comercialización; mientras que los perdedores suelen ser los consumidores finales, que no pueden adquirir los medicamentos a los precios de monopolio impuestos, ni acceder, por efecto de los derechos de exclusividad encarnados en las patentes, a medicamentos genéricos (Luna, 2012; Lindner, 2012). A esto último se agrega la regla 90-10, aquella que alude a que 90 por ciento de la investigación se dirige a enfermedades que afectan a 10 por ciento de la población (Stiglitz, 2010 citado en Berasaluce y Ruiz, 2012).

En cuarto lugar, el arbitraje internacional en materia de propiedad intelectual ha resultado ser un negocio bastante lucrativo para las grandes corporaciones y los bufetes de abogados especializados, en detrimento de los gobiernos demandados $\mathrm{y}$, a final de cuentas, del ciudadano común. Van Harten y Malysheuski (2016) estiman que los montos agregados en transferencias de los Estados demandados hacia los inversionistas fue de 9 mil 164 millones de dólares en 214 casos y 856 millones de dólares hacia la industria del arbitraje inversionista-Estado (véase diagrama 1). 
Diagrama 1. Ganancias de los arreglos en disputas inversionista-Estado (ISDS) (millones por caso adjudicado en paréntesis)

Grandes

ganadores

1. Compañías

extragrandes:

6 mil 526 millones

(136)

2. Industria

ISDS:

mil 712 millones

(8)

3. Individuos

súper ricos:

984 millones

(45)

4. Grandes

compañías:

628 millones (17)

\section{Ganadores \\ modestos}

5. Otros

individuos:

145 millones (3)

6. Compañías

medianas

y pequeñas:

36 millones (2)
Perdedores

modestos

7. Compañías con ingreso desconocido: 10 millones $(-0.2)$
Grandes

perdedores

8. Estados demandados: -10 mil 020 millones (-47)

Notas: 1) Ingreso anual $>10$ millones de dólares; 2) Abogados y mediadores.

3) Riqueza neta $>100$ millones de dólares de dólares. 4) 10 billones de dólares $>$ Ingreso anual $>1$ billón de dólares. 5) Riqueza neta $<100$ millones de dólares.

6) y 7) 1 billón de dólares $<$ Ingreso anual $<100$ millones de dólares.

Fuente: Van Harten y Malysheuski (2016:12-13).

Por último, y como efecto de las regulaciones internacionales referidas, muchos países son orillados a adherir agendas de investigación en ciencia y tecnología que devienen en consecuencias nefastas para sus economías y sociedades en cuestiones financieras, económicas y medioambientales. Van Harten (2011) alude a este fenómeno como una suerte de compra de soberanía por parte de las grandes corporaciones multinacionales. 


\section{Reflexiones finales}

La reestructuración de los sistemas de innovación constituye un mirador privilegiado para analizar y comprender el significado y las implicaciones de los modos de apropiación del conocimiento que distinguen a la globalización neoliberal y que subyacen a la lógica de dominación que acompaña a los TLC promovidos por las grandes corporaciones multinacionales y las principales potencias imperialistas, encabezadas por Estados Unidos. No son acuerdos en los que todos los participantes ganan, son estrategias que profundizan las dinámicas de desarrollo desigual que caracterizan al capitalismo contemporáneo. Conducen asimismo a una carrera desenfrenada hacia la expansión y apropiación de los productos del General Intellect bajo una perspectiva de obtención de ingentes ganancias monopólicas a cualquier costo.

Todo indica, en ese sentido, que nos encontramos ante una crisis, con visos de terminal, de la modernidad capitalista, que reclama avanzar hacia una modernidad alternativa, es decir, una modernidad no capitalista que «implique una verdadera abundancia y una verdadera emancipación (...) una modernidad que nunca fue, que nunca existió, no — como dice Habermas - completar el proyecto de modernidad, sino inventar otra distinta que fue posible incluso desde antes y que fue reprimida y negada, y hasta ahora postergada» (Echeverría, 2011:290). Se trata, en el fondo, de avanzar hacia una ruta de transformación social radical, centrada en las necesidades sociales y en armonía con la naturaleza, que entrañe, entre otros elementos, la defensa a ultranza de los bienes comunes tangibles e intangibles (Laval y Dardot, 2015). 


\section{Referencias}

Amin, Samir (2013), The Implosion of Capitalism, London, Pluto Press.

Andreff, Wladimir (2009), «Outsourcing in the New Strategy of Multinational Companies: Foreign Investment, International Subcontracting and Production Relocation», Papeles de Europa (18), pp. 5-34.

Bello, Walden (2005), Dilemmas of Domination: The Unmaking of the American Empire, New York, Metropolitan Books.

Berasaluce Iza, Julen y Salvador Francisco Ruiz Medrano (2012), «Patentes y otros incentivos a la innovación. Perspectiva económico-legal aplicada al caso de medicamentos», en Héctor Arturo Oropeza García y Víctor Manuel Guízar López (coords.), Los retos de la industria farmacéutica en el siglo XXI. Una visión comparada sobre su régimen de propiedad intelectual, México, Universidad Nacional Autónoma de México/Cofepris, pp. 351-376.

Borras, Saturnino, Jennifer Franco, Sergio Gómez, Cristóbal Kay \& Max Spoor (2012), «Land grabbing in Latin America and the Caribbean», The Journal of Peasant Studies, 39(3-4), pp. 845-872.

Brenner, Robert (2002), The Boom and the Bubble: The US in the World Econo$m y$, New York, Verso.

Delgado Wise, Raúl (2015), «Unraveling Mexican Highly-Skilled Migration in the Context of Neoliberal Globalization», in Stephen Castles, Magdalena Arias Cubas \& Derya Ozkul (eds.), Social Transformation and Migration: National and Local Experiences in South Korea, Turkey, Mexico and Australia, United Kingdom, Palgrave MacMillan, pp. 201-218.

Delgado Wise, Raúl \& David Martin (2015), «The political economy of global labour arbitrage», in Kees van der Pijl (ed.), The International Political Economy of Production, Cheltenham, Edward Elgar, pp. 59-75. 


\section{RAÚl DELGADO WISE}

Díaz, Álvaro (2008), América Latina y el Caribe: la propiedad intelectual después de los tratados de libre comercio, Santiago de Chile, Comisión Económica para América Latina y el Caribe.

Dobbs, Richard et al. (2012), The World at Work: Jobs, Pay, and Skills for 3.5 billion People, McKinsey Global Institute in http://www.mckinsey.com/ insights/employment_and_growth/the_world_at_work/19/03/2015 (2015), Playing to Win: The New Global Competition for Corporate Profits, en file://C:/Users/Raul/Downloads/MGI\%20Global\%20Competition_ Full\%20Report_Sep\%202015.pdf

Echeverría, Bolívar (2011), Antología. Crítica de la modernidad capitalista, La Paz, Oxfam/Vicepresidencia del Estado Plurinacional de Bolivia.

Emmanuel, Arghiri (1972), El intercambio desigual: ensayo sobre los antagonismos en las relaciones económicas internacionales, México, Siglo XXI.

Foladori, Guillermo (2014), «Ciencia ficticia», Estudios Críticos del Desarrollo 4(7), pp. 41-66.

Foster, John Bellamy (2010), «The Financialization of the Capitalist Class: Monopoly-Finance Capital and the New Contradictory Relations of Ruling Class Power», in Henry Veltmeyer (ed.), Imperialism, Crisis and Class Struggle: The Enduring Verities and Contemporary Face of Capitalism, Boston, Brill Publishers.

Foster, John Bellamy, Robert McChesney \& Jamil Jonna (2011a), «The Internationalization of Monopoly Capital», Monthly Review, 63(2), pp. 3-18. (2011b), «The Global Reserve Army of Labour and the New Imperialism», Monthly Review, 63(6), pp. 1-15.

Fulton, Deirdre (january 15th 2015), «Don't buy the hyphe: 20 years of data reveals 〈Free Trade〉 fallacies», Common Dreams, in http://www.common 
dreams.org/news/2015/01/15/dont-buy-hype-20-years-data-reveals-freetrade-fallacies

Galama, Titus \& James Hosek (2008), US Competitiveness in Science and Technology, Santa Mónica, Research and Development Corporation (RAND).

García Moreno, Víctor Carlos (1998), «El capítulo XVII del TLCAN y su influencia en la nueva Ley Mexicana del Derecho de Autor», en Manuel Becerra Ramírez (comp.), Estudios de derecho intelectual en homenaje al profesor David Rangel Medina, México, Universidad Nacional Autónoma de México, pp. 103-116.

Gereffi, Gary \& Timothy Sturgeon (2013), «Global value chain-oriented industrial policy: the role of emerging economies», in Deborah Elms \& Patrick Low (eds.), Global value chains in a changing world, Geneva, World Trade Organization, pp. 329-360.

Gudynas, Eduardo (2015), Extractivismos. Ecología, economía y política de un modo de entender el desarrollo y la naturaleza, Bolivia, Centro de Documentación e Información Bolivia.

Guerrero Castro, Rodrigo y Roberto Gutiérrez (2011), «Los ADPIC y el TLCAN en la industria farmacéutica mexicana: un análisis TradeCAN», Nueva Época (35), pp. 93-129.

Harvey, David (2014), Diecisiete contradicciones y el fin del capitalismo, Quito, Instituto de Altos Estudios Nacionales del Ecuador.

Laval, Christian y Pierre Dardot (2015), Común: ensayo sobre la revolución en el siglo XXI, Barcelona, Gedisa.

Luna, Alejandro (2012), «Patentes de invención. Patentes farmacéuticas, protección de datos clínicos y otros temas de interés para la industria farmacéutica en México», en Héctor Arturo Oropeza García y Víctor Manuel Guízar López (coords.), Los retos de la industria farmacéutica en el siglo XXI. Una 
visión comparada sobre su régimen de propiedad intelectual, México, Universidad Nacional Autónoma de México/Cofepris, pp. 391-424.

Márquez, Humberto (2010), «La gran crisis del capitalismo neoliberal», Andamios (13), pp. 57-84.

Messitte, Peter (2012), «Desarrollo del derecho de patentes estadounidense en el siglo XXI. Implicaciones para la industria farmacéutica», en Héctor Arturo Oropeza García y Víctor Manuel Guízar López (coords.), Los retos de la industria farmacéutica en el siglo XXI. Una visión comparada sobre su régimen de propiedad intelectual, México, Universidad Nacional Autónoma de México/Cofepris, pp. 179-200.

Miguelez, Ernest \& Carsten Fink (may 2013), «Measuring the international mobility of inventors: a new database», WIPO, Working Paper No. 8, en http:// www.wipo.int/edocs/pubdocs/en/wipo_pub_econstat_wp_8.pdf

Oxfam (2017), «Una economía para el 99\%. Es hora de construir una economía más humana y justa al servicio de las personas», en http://www.oxfammexico. org/wp-content/uploads/2017/01/bp-economy-for-99-percent-160117-es.pdf

Rodríguez Lascano, Sergio (2015), «Apuntes sobre el pensamiento crítico vs las mutaciones de la Hidra», en El Pensamiento Crítico Frente a la Hidra Capitalista II, México, EZLN, pp. 434-455.

Secretaría de Economía (6 noviembre 2015), «Capitulado completo del Tratado de Asociación Trans-Pacífico (TPP) versión español», en http://www.gob. $\mathrm{mx} / \mathrm{tpp} \#$ textos

Saxenian, AnnaLee (2002), Local and Global Networks of Immigrant Professionals in Silicon Valley, San Francisco, Public Policy Institute of California. (2006), The New Argonauts: Regional Advantage in a Global Economy, Boston, Harvard University Press. 
Shadlen, Kenneth (2011), «Propiedad intelectual para el desarrollo en México», en Kevin Gallagher, Enrique Dussel Peters y Timothy Wise (eds.), El futuro de la política de comercio en América del Norte: lecciones del TLCAN. Estados Unidos-México: Pardee Center Task Force Report/Universidad Autónoma de Zacatecas/Global Development and Environment Institute/Boston University/Frederick S. Pardee Center/Universidad Nacional Autónoma de México, en http://www.ase.tufts.edu/gdae/policy_research/pardee.html

Suwandi, Intan y John Bellamy Foster (2016), «Multinational Corporations and the Globalization of Monopoly Capital. From the 1960s to the Present», Monthly Review, 68(3).

United Nations Conference on Trade and Liberalization (UNCTAD) (2010), World Investment Report 2010, Nueva York, UNCTAD.

Van Harten, Gus \& Pavel Malysheuski (2016), «Who has benefited financially from investment treaty arbitration? An evaluation of the size and wealth of claimants», Osgoode Legal Studies Research Paper, 12(14).

Van Harten, Gus (2011), «Reformando el régimen de inversión del TLCAN», en Kevin Gallagher, Enrique Dussel Peters y Timothy Wise (eds.), El futuro de la política de comercio en América del Norte: lecciones del TLCAN. Estados Unidos-México: Pardee Center Task Force Report/Universidad Autónoma de Zacatecas/Global Development and Environment Institute/Boston University/Frederick S. Pardee Center/Universidad Nacional Autónoma de México, en http://www.ase.tufts.edu/gdae/policy_research/pardee.html Veltmeyer, Henry (2013), «The Political Economy of Natural Resource Extraction: A New Model or Extractive Imperialism?», Canadian Journal of Development Studies, 34(1), pp. 79-95. 


\section{RAúl Delgado Wise}

World International Property Organization (WIPO) (2017), «The PCT now has 152 Contracting States», in http://www.wipo.int/pct/en/pct_contracting_ states.html

Xing, Yuquing \& Charles Kolstad (2002), «Do Lax Environmental Regulations Attract Foreign Investment?», Environmental and Resource Economics, 21(1), pp. 1-22. 